\title{
LES PRÉFÉRENCES ALIMENTAIRES DU VEAU I. - APPÉTIBILITÉ COMPARÉE dES CÉRÉALES
}

PAR

\section{C.-M. MATHIEU et Erna WEGAT-LITRE}

avec la collaboration technique de P. Delhumeau et J. Lefaivre.

Station de Recherches sur l'Élevage, C. N. R. Z., Jouy-en-Josas (S.-et-O.).

\section{SOMMAIRE}

L'objet de cette étude a été de classer les principales céréales par ordre d'appétibilité, 1'orge étant prise comme céréale de référence.

Dans tous les essais, les céréales se sont classées dans le même ordre de préférence décroissante à savoir : orge, blé, seigle, maïs, avoine.

Il est intéressant de tenir compte de ces différences dans la préparation des aliments pour les veaux sevrés précocement.

Dans les régimes de sevrage précoce du veau on cherche à remplacer le lait par d'autres aliments, plus particulièrement par des concentrés distribués secs. Ces concentrés doivent être à la fois riches en éléments nutritifs et très appétibles, de façon à ce que le veau en consomme de $\mathrm{I}, 5$ à $2 \mathrm{~kg}$ par jour dès la fin du deuxième mois.

Un certain nombre d'auteurs ont étudié l'appétibilité des concentrés secs pour veaux (Mc CANdirish I923, SAVAgE et Crawrord I935, Newman et Savage i938, Norton et Faton i946, Preston i956, Miller, Carmon et Dalton r958 et r959). En fait, ils se sont attachés à l'étude d'un seul concentré en vue de le rendre appétible par approximations successives. I,eurs résultats sont très fragmentaires et ne nous renseignent que sur un petit nombre d'aliments (tourteau de soja, d'arachide, levures) et sur l'influence du mode de présentation.

Par contre, aucun auteur ne semble avoir fait une étude systématique de l'appétibilité, laquelle doit comporter successivement : teaux...),

a) 1'étude de l'appétibilité des aliments simples (céréales, tour- 
b) l'influence du mode d'association des aliments

c) l'influence du mode de préparation et de présentation.

Dans ce programme général nous avons commencé par étudier l'appétibilité des principales céréales. Celles-ci représentent en effet la fraction la plus importante des aliments concentrés du veau $(60$ à 80 p. IOO).

\section{MATÉRIEL ET MÉTHODES}

I'appétibilité étant une qualité relative, elle doit être déterminée par rapport à un aliment de référence. Dans le cas des céréales nous avons choisi l'orge comme base par analogie avec le système des unités fourragères.

Dans chacun des essais nous avons offert simultanément aux veaux le concentré de référence à base d'orge et le concentré expérimental contenant la céréale étudiée: avoine, blé, maìs, seigle. Nous avons mesuré les quantités consommées de chacun des concentrés et exprimé l'appétibilité (WIDMaRK I944) par le rapport :

$$
\text { Ioo quantité consommée du concentré expérimental }
$$

Dans nos essais nous avons limité la quantité totale de lait consommé à 2001 par tête, avec un sevrage à sept semaines, ceci pour trois raisons :

- C'est la quantité minimum compatible avec une croissance satisfaisante pour les bouvillons et pour les génisses d'ólevage.

- Cette limitation permet de stimuler précocement la consommation de concentré et de mettre en évidence les variations de cette conscmmation.

- C'est dans ces régimes comportant une quantité de lait aussi restreinte, que se pose vraiment le problème de l'appétibilité des concentrés.

Dans un tel régime le veau ne peut pas se contenter de céréales seules; il doit recevoir un concentré qui contienne environ $200 \mathrm{~g}$ de matières azotées digestibles par kilogramme. Nous l'avons préparé en adjoignant à deux parties de céréales une partie d'un mélange azoté, le tout étant présenté sous forme de mouture grossière. Afin de préciser si le mode d'association de la céréale avec un autre aliment modifiait son appétibilité, deux mélanges azotés ont été utilisés : un sans et un avec farine de poisson (tableau I).

Nous avons réalisé ces essais d'une part sur des veaux élevés en lots (essai I) et, d'autre part, sur des veaux élevés en cases individuelles (essai 2). Le tableau II récapitule les II essais qui ont été effectués. 
TABLEAU I

Composition des compléments azotés mélangés aux céréales.

\begin{tabular}{|c|c|c|}
\hline & $\begin{array}{c}\text { Mélange sans farine } \\
\text { de poisson }\end{array}$ & $\begin{array}{c}\text { Mélange avec farine } \\
\text { de poisson }\end{array}$ \\
\hline 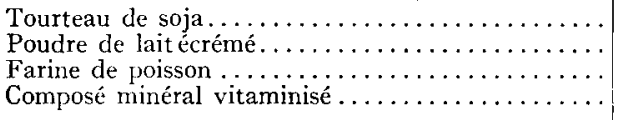 & $\begin{array}{l}60 \% \\
30 \% \\
10 \%\end{array}$ & $\begin{array}{l}60 \% \\
\text { I5 \% } \\
15 \% \\
10 \%\end{array}$ \\
\hline Matières azotées digestibles...... & $35 \%$ & $39 \%$ \\
\hline
\end{tabular}

\section{TABLEAU II}

Tableau récapitulatif des essais effectués.

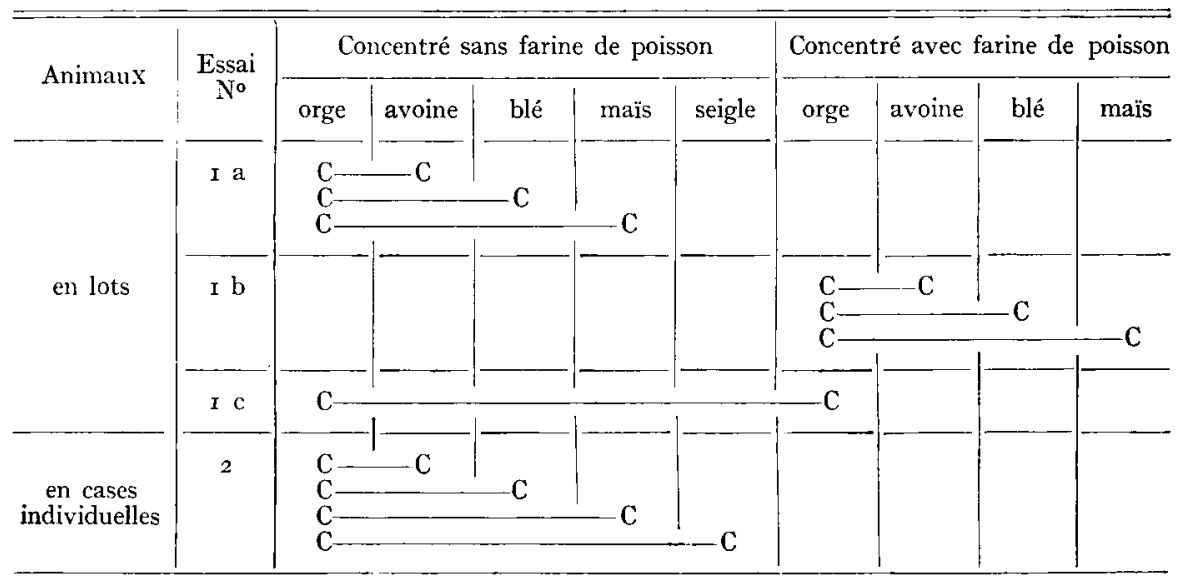

(La lettre " $\mathrm{C}$ " reqrésente le concentré donné. Les traits horizontaux schématisent la distribution simultanée des deux concentrés " $\mathrm{C}-\mathrm{C}$ \%)

Les deux compléments azotés ont été utilisés dans les essais I en lot (essai I $a$ sans farine de poisson et I $b$ avec farine de poisson). Un lot supplémentaire (essai I $c$ ) a reçu de l'orge avec chacun des deux compléments azotés.

Dans les essais individuels (essai 2) seul le complément sans farine de poisson a été employé.

\section{Réalisation des mesures}

\section{Animaux.}

Pour les essais en lots nous avons acheté des veaux normands âgés de huit jours et avons constitué des groupes de 4 à Io veaux, aussi semblables que possible; cependant la proportion des mâles et des femelles, 
ainsi que le poids moyen des lots n'ont pas toujours été identiques en raison de l'échelonnement des achats. Les veaux alimentés individuellement étaient des veaux mâles de race Charolaise, Française Frisonne Pie Noire, Normande et Pie Rouge de 1'Est. Ils furent appariés au point de vue race, poids et âge.

Nous avons utilisé au total Io2 veaux, dont les tableaux IV et $\mathrm{V}$ donnent la répartition entre les différents essais. Huit veaux sont morts en cours d'expérience. Cinq de ces décès ont été attribués à des œđèmes pulmonaires occasionnés par l'ingestion de farines trop fines présentées en cours d'essai. C'est pourquoi 4 essais ont été répétés sur des lots supplémentaires.

\section{Alimentation.}

Le lait a été distribué individuellement deux fois par jour à la température de $35^{\circ} \mathrm{C}$. De la naissance au sevrage (sept semaines) chaque veau a consommé 2001 de lait entier, répartis de la façon suivante : $4,5,6,5,4,3,2$ litres par jour respectivement pour chacune des sept premières semaines.

Le concentré a été distribué une fois par jour, que ce soit en lot ou individuellement. I,es rations au cours des premières semaines des essais furent de $0,5 \mathrm{~kg}$ par jour et par tête, puis elles augmentèrent par paliers de $0,5 \mathrm{~kg}(0,5 \mathrm{~kg}, \mathrm{I} \mathrm{kg}, \mathrm{I}, 5 \mathrm{~kg} .$.$) de façon à ce que les animaux en aient$ toujours à volonté. Le changement de palier a été effectué, quand les refus n'étaient plus que de ro à 20 p. Ioo de la quantité offerte. Les refus ont été pesés chaque matin.

Pour éviter le conditionnement à l'emplacement (AschkenasyLELU, I95I) nous avons intervertilles auges de concentré au moins une fois par semaine.

\section{TABLEAU III}

Caractéristiques des concentrés et du toin utilisés.

\begin{tabular}{|c|c|c|c|c|c|c|}
\hline & Céréales & $\begin{array}{c}\text { Matière } \\
\text { sèche } \\
\%\end{array}$ & $\begin{array}{c}\text { Unités } \\
\text { fourragères } \\
\text { par kg }\end{array}$ & $\begin{array}{c}\text { Matières } \\
\text { azotées } \\
\%\end{array}$ & $\begin{array}{c}\text { Matières } \\
\text { grasses } \\
\%\end{array}$ & $\begin{array}{c}\text { Matières } \\
\text { cellulosiques } \\
\%\end{array}$ \\
\hline $\begin{array}{l}\text { Concentrés } \\
\text { avec farine } \\
\text { de poisson }\end{array}$ & $\begin{array}{l}\text { Orge ..... } \\
\text { Avoine ... } \\
\text { Blé ...... } \\
\text { Mais ...... }\end{array}$ & $\begin{array}{l}82,9 \\
90,5 \\
82,3 \\
85,4\end{array}$ & $\begin{array}{l}0,99 \\
0,86 \\
1,00 \\
1,05\end{array}$ & $\begin{array}{l}19,6 \\
21,7 \\
20,8 \\
20,3\end{array}$ & $\begin{array}{l}3,1 \\
5,0 \\
2,8 \\
4,0\end{array}$ & $\begin{array}{l}4,0 \\
8,8 \\
2,4 \\
2,3\end{array}$ \\
\hline $\begin{array}{l}\text { Concentrés } \\
\text { sans farine } \\
\text { de poisson }\end{array}$ & $\begin{array}{l}\text { Orge ...... } \\
\text { Avoine .... } \\
\text { Blé ..... } \\
\text { Maïs ..... } \\
\text { Seigle .... }\end{array}$ & $\begin{array}{l}85,7 \\
87, \mathrm{I} \\
85, \mathrm{I} \\
85,5 \\
86,8\end{array}$ & $\begin{array}{l}0,95 \\
0,83 \\
1,00 \\
1,04 \\
1,02\end{array}$ & $\begin{array}{l}19,3 \\
18,9 \\
21,3 \\
18,6 \\
20,4\end{array}$ & $\begin{array}{l}2,6 \\
4,3 \\
2,3 \\
3,6 \\
2,0\end{array}$ & $\begin{array}{l}4,9 \\
8,3 \\
3,1 \\
2,9 \\
3,3\end{array}$ \\
\hline \multicolumn{2}{|c|}{ Foin à base de dactyle ... } & 91,8 & 0,48 & 9,3 & 4,8 & 32,3 \\
\hline
\end{tabular}


Un bon foin à base de dactyle a été distribué à volonté deux fois par jour. Les refus n'ont été pesés qu'une fois, le mati:.

Les caractéristiques des concentrés et du foin utilisés sont résumées dans le tableau III.

Les consommations de concentré ont été enregistrées chaque jour de la troisième à la douzième semaine d'âge. Elles concernent donc une période de neuf semaines, dont quatre précédant le sevrage.

\section{RÉSULTATS}

\section{I $^{\mathrm{o}}$ Essais en lots.}

Le tableau IV donne les résultats globaux et la consommation de chacun des concentrés exprimée en pourcentage de la consommation totale de concentré.

\section{TABLEAU IV}

Résultats des essais I (veaux en lots).

\begin{tabular}{|c|c|c|c|c|c|c|c|c|}
\hline \multirow{3}{*}{$\begin{array}{c}\text { Essai } \\
n^{0}\end{array}$} & \multirow{3}{*}{$\begin{array}{l}\text { Céréales } \\
\text { comparées }\end{array}$} & \multirow{3}{*}{$\begin{array}{l}\text { Nom- } \\
\text { bre de } \\
\text { veaux }\end{array}$} & \multirow{2}{*}{\multicolumn{2}{|c|}{$\begin{array}{l}\text { Quantités totales } \\
\text { d'aliments consommées } \\
\text { par veau de la } 4^{\mathrm{e}} \\
\text { a la } \mathrm{I} \mathbf{2}^{\mathrm{e}} \text { semaine }\end{array}$}} & \multicolumn{4}{|c|}{$\begin{array}{l}\text { Consommation en } \% \text { de la } \\
\text { consommation totale de concentré }\end{array}$} \\
\hline & & & & & \multicolumn{2}{|c|}{$\begin{array}{l}\text { Sans farine } \\
\text { de poisson }\end{array}$} & \multicolumn{2}{|c|}{$\begin{array}{l}\text { Avec farine } \\
\text { de poisson }\end{array}$} \\
\hline & & & Concentré & Foin & Orge & $\begin{array}{l}\text { Céréale } \\
\text { étudiée }\end{array}$ & Orge & $\begin{array}{l}\text { Céréale } \\
\text { étudiée }\end{array}$ \\
\hline I $a$ & $\begin{array}{l}\text { Orge/Avoine ... } \\
\text { Orge/Blé....... } \\
\text { Orge/Maîs .... }\end{array}$ & $\begin{array}{r}7 \\
10 \\
18\end{array}$ & $\begin{array}{l}32,9 \\
5,9,0 \\
66,8\end{array}$ & $\begin{array}{l}32,9 \\
14,6 \\
15,2\end{array}$ & $\begin{array}{l}66 \\
66 \\
68\end{array}$ & $\begin{array}{l}34 \\
34 \\
3^{2}\end{array}$ & & \\
\hline I $b$ & $\begin{array}{l}\text { Orge/Avoine ... } \\
\text { Orge/Blé... . . . } \\
\text { Orge/Maïs ... }\end{array}$ & $\begin{array}{r}10 \\
12 \\
9\end{array}$ & $\begin{array}{l}62,5 \\
69,2 \\
4,3,2\end{array}$ & $\begin{array}{l}2,3,5 \\
30,8 \\
\mathrm{I} 2,7\end{array}$ & & & $\begin{array}{l}95 \\
54 \\
80\end{array}$ & $\begin{array}{r}5 \\
46 \\
20\end{array}$ \\
\hline I $c$ & Orge/Orge .... & 6 & 51,6 & 7,0 & 74 & & 26 & \\
\hline
\end{tabular}

L'orge a été préférée aux autres céréales dans les deux essais I a et I $b$ (avec et sans farine de poisson).

D'autre part, si l'essai I a ne permet pas de différencier nettement l'appétibilité des autres céréales, l'essai I $b$ permet de préciser la gamme de leur appétibilité, qui s'établit comme suit, par ordre décroissant de préférence : orge, blé, maïs, avoine. La présence de la farine de poisson semble donc avoir révélé les différences d'appétibilité entre les céréales (essais I $a$ et I $b$ ).

Des deux concentrés de référence à base d'orge, l'un avec, l'autre sans farine de poisson, le plus appétible est nettement celui sans farine de poisson (Essai I c). 

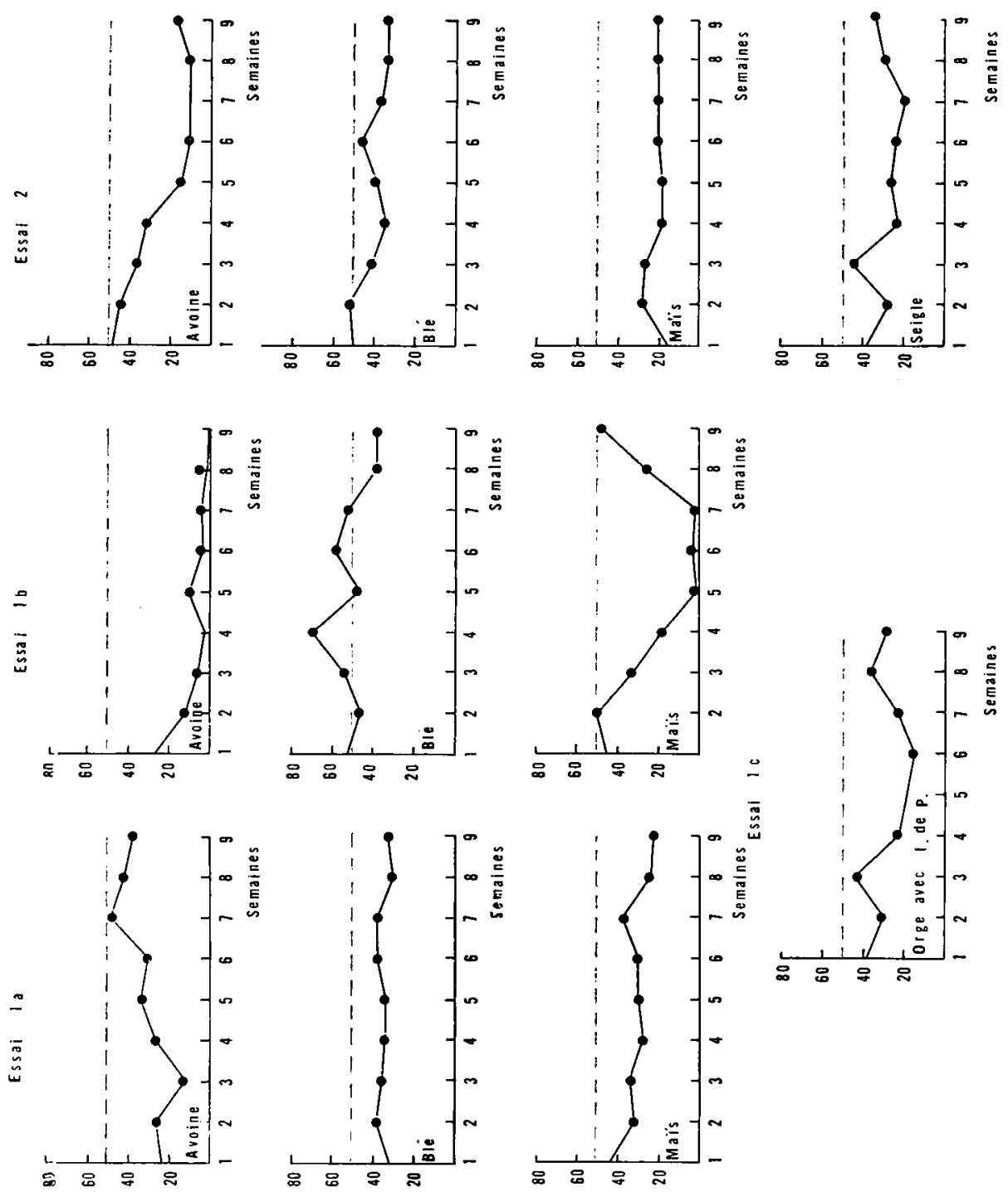

Grapiligue: I. - Evolution de la consommation du concentré expérimental pour chacune des neuf semaines de l'expérience (en pourcentage de la consommation totale de concentré).

$I^{\circ}$ Essais en lot :

I $a$ Concentré sans farine de poisson.

I $b$ Concentré avec farine de poisson.

I $c$ Deux concentrés de référence (orge sans et avec farine de poisson).

$2^{0}$ Essai en cases individuelles :

Concentré sans farine de poisson. 
Si nous scindons les consommations pour les répartir en deux périodes, avant le sevrage ( 4 semaines) et après le sevrage ( 5 semaines), nous constatons que le classement des céréales est le même dans les deux périodes. De même le graphique $I$, donnant par semaine la proportion du concentré expérimental consommée par rapport à la consommation totale de concentré, montre que les rapports des quantités consommées de concentré pour un même lot sont demeurés relativement constants. Il semble donc, que les veaux aient fait un choix rapide et définitif.

Les quantités globales consonmées sont différentes d'un lot à l'autre en raison du manque de similitude des lots et des différences dans la qualité du foin. Nous avons été obligés de distribuer un foin âgé en fin d'expérience (avril et mai) pour certains essais ( $c$ par exemple).

\section{$2^{\circ}$ Essai en cases individuelles.}

La méthode en lot, utilisée dans les essais précédents, pouvait masquer les préférences alimentaires inhérentes à l'individu. A titre de contrôle et pour obvier à cet inconvénient des essais ont été repris en cases individuelles.

Un des essais précédents (I $c$ ) ayant montré la faible appétibilité de la farine de poisson, nous n'avons utilisé que le complément azoté sans farine de poisson pour cet essai. Aux céréales déjà étudiées, avoine, blé, mais, orge, le seigle a été ajouté.

Les sujets de cet essai étaient de quatre races différentes (Charolaise, Irrançaise İrisonne Pie Noire, Nornande, Pie Rouge de 1'Esst); de plus ils étaient logés dans deux étables distantes de $7 \mathrm{~km}$. Chaque lot était constitué de 8 veaux, un de chacune des quatre races dans chacune des deux étables.

Les résultats individuels sont reportés au tableau VI et les moyennes au tableau V. Ils établissent le même classement d'appétibilité que les essais en lot, ’̀ savoir : orge, blé, seigle, naïs, avoine. Le seigle prend place entre le blé et le maïs. De même les veaux ont effectué un choix rapide et définitif (graphique I).
TABLEAL $Y$
Résultats de l'essai 2 (veaux en cases individuelles).

\begin{tabular}{|c|c|c|c|c|c|}
\hline \multirow[t]{2}{*}{ Céréales comparées } & \multirow{2}{*}{$\begin{array}{c}\text { Nombre } \\
\text { de } \\
\text { veaux }\end{array}$} & \multicolumn{2}{|c|}{$\begin{array}{l}\text { Quantités totales d'aliments } \\
\text { consommées par veau } \\
\text { de la } 4^{\mathrm{e}} \dot{\mathrm{a}} \text { la } 12^{\mathrm{e}} \text { semaine }\end{array}$} & \multicolumn{2}{|c|}{$\begin{array}{l}\text { Consommation en } \% \text { de la } \\
\text { consommation totale de } \\
\text { concentré }\end{array}$} \\
\hline & & Concentrés & Foin & Orge & Céréale étudiée \\
\hline Orge : Avoine. & 8 & $5^{6,3}$ & $16, \mathrm{I}$ & 84 & 16 \\
\hline Orge / Blé.... & 7 & 88,0 & 20,4 & 67 & 33 \\
\hline Orge / Mais... & 8 & 74,2 & $16, \mathrm{I}$ & 78 & $\begin{array}{l}33 \\
22\end{array}$ \\
\hline Orge / Seigle.. & 7 & 58,4 & 15,7 & 73 & 27 \\
\hline
\end{tabular}


Tous les individus ont eu les mêmes préférences indépendamment de la race. Par contre les différences d'appétibilité ont été beaucoup plus marquées dans l'étable I que dans l'étable 2.

TABLEAAU VI

Résultats de l'essai 2 : résultats individuels.

\begin{tabular}{|c|c|c|c|c|c|c|c|}
\hline \multirow{3}{*}{$\begin{array}{c}\text { Céréales } \\
\text { comparées }\end{array}$} & \multirow{3}{*}{ Race } & \multicolumn{6}{|c|}{ Consommation en p. too de la consommation totale de concentré } \\
\hline & & \multicolumn{3}{|c|}{ Étable I } & \multicolumn{3}{|c|}{ Étable 2} \\
\hline & & Orge & $\begin{array}{l}\text { Céréale } \\
\text { étudiée }\end{array}$ & $\begin{array}{l}\text { Écart jar } \\
\text { rapport } \\
\text { a la } \\
\text { moyenne }\end{array}$ & Orge & $\begin{array}{l}\text { Céréale } \\
\text { étudiée }\end{array}$ & $\begin{array}{l}\text { Ecart par } \\
\text { rapport } \\
\text { à la } \\
\text { moyenne }\end{array}$ \\
\hline Orge/avoine & $\begin{array}{l}\text { Charolaise . } \\
\text { Frisonne ... } \\
\text { Normande.. } \\
\text { Pie-Rouge . }\end{array}$ & $\begin{array}{l}86,6 \\
93,0 \\
93,8 \\
98,4\end{array}$ & $\begin{array}{r}11,4 \\
7,0 \\
6,2 \\
1,6\end{array}$ & $\begin{array}{l}4,8 \\
0,4 \\
0,4 \\
5,0\end{array}$ & $\begin{array}{l}69,7 \\
71,8 \\
75,9 \\
73,8\end{array}$ & $\begin{array}{l}30,3 \\
28,2 \\
24,1 \\
26,2\end{array}$ & $\begin{array}{l}3,1 \\
I, 0 \\
3, I \\
I, 0\end{array}$ \\
\hline Moyenne. & & 93,4 & 6,6 & 2,6 & 72,8 & 27,2 & 2,0 \\
\hline Orge/Blé & $\begin{array}{l}\text { Charolaise . } \\
\text { Frisonne ... } \\
\text { Normande.. } \\
\text { Pie-Rouge . }\end{array}$ & $\begin{array}{l}88,4 \\
79,4 \\
79,8 \\
7 \mathrm{I}, 4\end{array}$ & $\begin{array}{l}\text { I I } 6 \\
20,6 \\
20,2 \\
28,6\end{array}$ & $\begin{array}{l}8,7 \\
0,3 \\
0,1 \\
8,3\end{array}$ & $\begin{array}{l}\overline{48,} \\
54,7 \\
43,4\end{array}$ & $\begin{array}{l}51,9 \\
45,3 \\
56,6\end{array}$ & $\begin{array}{l}- \\
0,6 \\
6,0 \\
5,3\end{array}$ \\
\hline Moyenne & & 79,7 & 20,3 & 4,3 & 48,7 & 51,3 & 4,0 \\
\hline Orge/Maïs & $\begin{array}{l}\text { Charolaise } \\
\text { Frisonne ... } \\
\text { Normande.. } \\
\text { Pie-Rouge . }\end{array}$ & $\begin{array}{l}9 \mathrm{I}, \mathrm{I} \\
93,4 \\
9 \mathrm{I}, 5 \\
94, \mathrm{I}\end{array}$ & $\begin{array}{l}8,9 \\
6,6 \\
8,5 \\
5,9\end{array}$ & $\begin{array}{l}\mathrm{I}, 4 \\
0,9 \\
\mathrm{I}, 0 \\
\mathrm{I}, 6\end{array}$ & $\begin{array}{l}84,6 \\
56,0 \\
61,2 \\
6,3,7\end{array}$ & $\begin{array}{l}\text { I } 5,4 \\
44,0 \\
38,8 \\
36,3\end{array}$ & $\begin{array}{r}\mathrm{I} 8,3 \\
\mathrm{I} 0,3 \\
5, \mathrm{I} \\
2,5\end{array}$ \\
\hline Moyenne & & 92,5 & 7,5 & $\mathrm{I}, 2$ & 66,3 & 33,7 & 9,1 \\
\hline Orge/seigle & $\begin{array}{l}\text { Charolaise . } \\
\text { Frisonne ... } \\
\text { Normande.. } \\
\text { Pie-Rouge . }\end{array}$ & $\begin{array}{l}92,0 \\
84,0 \\
62,7 \\
68,7\end{array}$ & $\begin{array}{r}8,0 \\
16,0 \\
37,3 \\
31,3\end{array}$ & $\begin{array}{r}15,2 \\
7,2 \\
14, \mathrm{I} \\
8, \mathrm{I}\end{array}$ & $\begin{array}{c}81,9 \\
63,7 \\
60,3 \\
-\end{array}$ & $\begin{array}{l}18,1 \\
36,3 \\
39,7 \\
-\end{array}$ & $\begin{array}{r}13,3 \\
4,9 \\
8,3 \\
-\end{array}$ \\
\hline Moyenne & & 76,8 & 23,2 & $1 \mathrm{I}, \mathrm{I}$ & 68,6 & $3 \mathrm{I}, 4$ & 8,8 \\
\hline
\end{tabular}

\section{DISCUSSION}

Pour comparer l'appétibilité d'une céréale à celle de l'orge prise comme référence, nous avions le choix entre deux méthodes. Dans la première, nous offrons simultanément les deux aliments à chacun des veaux, qui a ainsi la possibilité de choisir. Dans la deuxième nous offrons l'aliment expérimental à un lot d'animaux et l'aliment de référence à un autre lot. Nous avons retenu la première pour deux raisons; d'abord elle n'implique pas de constituer des lots semblables, ce qui permet de diminuer le nombre d'animaux nécessaires; ensuite elle amplifie les différences d'appétibilité puisque l'animal peut choisir, alors que dans le cas de la deuxième méthode il est obligé de consommer pour satisfaire son appétit. 
La gamme d'appétibilité ainsi obtenue : orge, blé, seigle, maïs, avoine a été indépendante :

- du mode de distribution (en lot ou individuellement),

- du lieu d'expérience (deux étables),

- de la race des animatix (quatre races étudiées),

- de la nature du complément azoté (avec et sans farine de poisson),

- de J'âge des animaux (graphique I).

Elle doit donc traduire des différences d'appétibilité intrinsèques entre les céréales, dont nous n'avons pas analysé les causes. Il est difficile de les expliquer par des différences dans la composition chimique déterminée suivant les méthodes classiques (tableau III). I1 faut noter cependant que les céréales les moins appréciées (avoine, maïs) se trouvent être les plus riches en matières grasses.

I,e degré d'appétibilité d'une céréale donnée peut être modifié par certains facteurs extrinsèques.

L'addition de la farine de poisson a accentué les différences observées (tableau IV). C'est ainsi qu'elle a rendu l'avoine pratiquement inappétible. Il s'agit là probablement d'un phénomène d'appétibilité associative.

Le lieu a aussi modifié les degrés d'appétibilité (tableau VI). Dans l'étable I la supériorité de l'orge est systématiquement plus accentuée que dans 1'étable 2 : dans cette dernière orge et blé ont même présenté une appétibilité très voisine. Ces différences sont très difficiles à interpréter ; elles peuvent être dues aux différences dans l'environnement, dans la qualité du lait, dans la disposition des cases...

Ce classement des céréales par ordre d'appétibilité diffère de celui généralement admis, et suivant lequel le mais et surtout l'avoine sont les céréales les plus appréciées par le veau.

Notons aussi, que l'essai Ic a montré, que le veau préférait la poudre de lait écrémé à la farine de poisson.

\section{SUMMARY}

Io The object of this study was to classify the main cereals : oats, wheat, maize, barley, rye, in order of their palatability for calves. In each of the trials (table II) the calves received, simultaneously, the control calf starter, based on barley, and the experimental calf starter containing the cereal studied.

$2^{\circ}$ One hundred and two calves, some in groups and some fed individually, were used in the experiments. The calves received, in addition to two calf starters available at choice, 2001 of whole milk, hay, and water ad lib.

$3^{\circ}$ In all the trials, the barley was the most palatable of the five cereals studied. The others are classed in decreasing order : wheat, rye, maize and oats.

The fish meal given in one of the protein supplements was found to be much less palatable that skim milk powder. 
$4^{\circ}$ This scale of palatability remained the same irrespective of :

a) the method of distribution;

b) the experimental stables;

c) the breed of animal;

d) the age of the animals;

c) the nature of the protein supplements.

It seems fo be there some intrinsic differences between the cereals.

Nevertheless some different degrees of palatability related to certain extrinsic factors (presence of fish meal, stable) were apparent (table IV andVI).

\section{RÉFÉRENCES BIBLIOGRAPHIQUES}

Aschkenasy-Leidu P., I95I. L'ingestion alimentaire en fonction de la composition du régime. Application à la régulation du besoin azoté chez le rat. Ann. Nutr. Alim., 5, 453-48r.

McCandist A. C., I923. Studies in the Growth and the Nutrition of Dairy Calves. J. Dairy Sci, 6, 500.

Miliek W. J., Carmon J. L. et Dalton H. L., I958. Influence of Anise Oils on the Palatability of Calf Starters. J. Dairy Sci., 41, I262.

Miller W. J., Carmon J. L. et Dalton H. L., I959. Influence of High Levels of Plant and Animal Fats in Calf Starters on Growth, Feed Consumption and Palatability. J. Dairy Sci., 42, I53.

Newman P. E. et Savage E. S., I938. The use of Yeast in Calf Meals and Pellets. J. Dairy Sci., 21, I6I.

Norton C. L. et Eaton H. D., I946. Dry Calf Starters for Dairy Calves. Cornell Agr. Exp. Sta. Bu1l. 835, 32 pages.

PRESTON T. R., I956. Studies on the Raising of Calves Weaned from Milk between two and four Weeks of Age. Proceed. Brit. Soc. Anim. Prod., p. $67-77$.

SAVAge E. S. et Crawford C. H., I935. Dry Concentrates as a Partial Substitute for Whole Milk in Calf Rations. Cornell Agr. Exp. Sta. Bull. 622,29 pages.

Widmark E. M. P., r944. The Selection of Food. I. General Consideration and Methodical Notes. Acta Physiol. Scand., 7, I47-I54. 